\title{
SESSION H:
}

\section{EMP PHENOMENA}


US Army Electronics Research and Development Command

Harry Diamond Laboratories

2800 Powder Mill Road

Adel phi, Maryland 20783

\section{ABSTRACT}

Ionization chamber measurements of bulk ionized air conductivity are complicated by the formation of a diode-like electron depletion layer at the interface of the negatively charged cathode and the ionized gas. Conduction across the electron depletion layer is dominated by ionic conductivity. The production of secondary electrons at the cathode by positive ion bombardment is postulated to explain abnormally large currents across the electron depletion layer at late time in $\mathrm{N}_{2}$ gas. A biased waveguide $\mathrm{rf}$ absorption technique has been developed to measure the electron conductivity of ionized gases in the region outside the electron depletion region.

\section{INTRODUCTION}

It would be very satisfying if a consistent set of microscopic ionized air chemistry parameters such as electron-molecule collision frequencies, electron attachment rates, and ionic recombination rates were available from which the macroscopic constitutive parameters of ionized air, such as air conductivity, could be derived. It would be even more satisfying if the microscopic parameters derived from the macroscopic measurements were consistent with microscopic experimental measurements such as electron scattering cross section measurements, etc. Typically, however, this is not the case, and it is, therefore, not surprising that the air chemistry packages used by workers in the EMP community, which to a large extent are based on microscopic parameters, do not always yield the same values of air conductivity. The work presented here is part of an ongoing program at HDL to develop the analytical and experimental tools necessary to correct some of the deficiencies in our knowledge of these basic air chemistry parameters.

The experiments reported here not only provide information about reaction rates, but also lead to information about boundary layers and conduction across these boundary layers. An understanding of boundary layers is necessary if bulk measurements are to be related to microscopie parameters.

Macroscopic ionized air conductivity measurements have in the past been made using a "pie-pan" ionization chamber. ${ }^{1-4}$ Figure 1 shows a schematic diagram of a "pie-pan" ionization chamber. Electrons are easily absorbed by a metallic conductor but, due to the metallic work function, it is difficult for an electron to pass from a negatively charged metallic plate to an ionized gas at ordinary temperatures. The field emission of electrons from a metallic electrode at room temperature requires a very large electric field ${ }^{5}$ and the theoretical interpretation of field emission data is complicated by the formation of local "hot spots".6,7 The electrons in the ionized gas migrate away from the negatively charged metallic electrode resulting in an electron depletion layer at the interface of the negatively charged metallic electrode and the ionized gas. The electric field in this region increases significantly, decreasing with distance from the negatively charged metal surface. Fig- ure 2 is a schematic diagram of the electron depletion layer at the interface of a negatively charged metallic conductor and an ionized gas.

We examined the diode-like behavior of an interface between an ionized gas and a metallic conductor at very high electric fields. Our experimental results cannot be accounted for by simple field emission. Because of the complexity of depletion layer effects, we developed biased waveguide techniques to obtain more insight into the macroscopic behavior of ionized gases, such as ionized air.

\section{THEORETICAL DISCUSSION OF PIE-PAN IONIZATION CHAMBERS}

The variation of ion and electron density in the electron-depletion region at the interface of a negatively charged metal and a collision-dominated ionized gas is described by equations 1 to $3 .^{1}$

$$
\begin{aligned}
& \frac{\partial N_{e}}{\partial t}+\frac{\partial V_{e} N_{e}}{\partial X}=S-\left(\alpha-G+\beta N_{+}\right) N_{e} \\
& \frac{\partial N_{+}}{\partial t}+\frac{\partial V_{+} N_{+}}{\partial X}=S+G N_{e}-\left(\beta N_{e}+\gamma N_{-}\right) N_{+} \\
& \frac{\partial N_{-}}{\partial t}+\frac{\partial V_{-} N_{-}}{\partial X}=\alpha N_{e}-\gamma N_{+} N_{-}
\end{aligned}
$$

where

$$
\begin{aligned}
& N_{+}=N_{e}+N_{-} \\
& S^{-}=i o n \text { pair production rate, } \\
& \alpha=\text { attachment rate coefficient, } \\
& G=\text { avalanche rate coefficient, } \\
& B=\text { electron-ion recombination coefficient, } \\
& \gamma=\text { ion-ion recombination coefficient, } \\
& N_{e}=\text { electron density, } \\
& N_{+}=\text {positive ion density, } \\
& N_{-}^{+}=\text {negative ion density, }
\end{aligned}
$$

and

$$
\mathrm{V}_{\mathrm{x}}=\text { electron or ion velocity. }
$$

Equations 1 to 3 can be combined with a suitable airchemistry package to calculate the development of the electron-depletion layer in an ionized gas. ${ }^{3,8-10}$

A difficulty that arises in the interpretation of pie-pan ionization chamber air conductivity measurements is that the behavior of the depletion layer is not thoroughly understood when the electric field in this region becomes very large. In order to gain a better understanding of the phenomena involved, measurements were done with both ambient air and nitrogen. Since nitrogen is non-attaching, boundary layer effects are amplified, while electron attachment in air tends to minimize them. In figure 3, the measured current response of an air-filled, pie-pan ionization chamber in the Aurora flash $x$-ray environment is compared with calculated current responses. The theoretical calculations are based on equations 1 to 3 for a number of different air chemistry packages. In figure 4 , the response of a nitrogen filled pie-pan (at 
Aurora) is compared to the response prediction. The agreement between the experimental results and calculation is much better for the ionized air chamber than for the ionized nitrogen chamber. The explanation lies in the fact that air is a mixture of nitrogen and oxygen. The behavior of the ionized air conductivity measurement at late time (at a time when the Aurora bremsstrahlung pulse has terminated) is dominated by ionic conductivity because the conduction electrons produced by the burst of bremsstrahlung quickly attach to $\mathrm{O}_{2}$ molecules $\left(10^{-8} \mathrm{sec}\right.$ attachment time). Since electrons do not readily attach to $\mathrm{N}_{2}$, the late-time behavior of ionized nitrogen is dominated by electronic conductivity. One would expect that the mitigating effects associated with the metallic work function would terminate the electron flow once the ionizing radiation was terminated. However, the experimental data shown in figure 4 suggest that electrons are actually leaving the metal surface. If this were not the case, then the late time current would decrease much faster as indicated by the dotted line.

A possible explanation for the deviation between prediction and measurement in the case of the $\mathrm{N}_{2}$ measurements is the production of secondary electrons resulting from ionic impact at the interface between the depletion layer and the negatively charged metallic cathode.11-18 According to equations 1-3, the electric field intensity at the interface between the cathode and the ionized gas when the pie-pan is biased to 1000 volts is $3.2 \times 10^{6}$ volts per meter. If the mean free path of an ion between collisions at atmospheric pressure is assumed to be about $10^{-5} \mathrm{~cm}$, a positive ion impinging on the cathode can have an energy up to 32 electron volts.

Figures 5 and 6 show comparisons between pie-pan current measurements and theoretical calculations for various probabilities of secondary electron emission due to $\mathrm{N}_{2}^{+}$ions impinging on the cathode. A 0.5 percent probability of electron emission yields a good fit to the experimental results.

The authors know of no data for secondary electron emission for $\mathrm{N}_{2}^{+}$ions incident on aluminum; however, data for $\mathrm{Ta}$ and $\mathrm{Pt}$ surfaces does exist.12,13 The se data are not inconsistent with an 0.5 percent probability for electron secondary emission due to ionic impact.

The pie-pan ionization chamber employed in this work had a circular area of $5 \mathrm{~cm}^{2}$ and a spacing between electrodes of $0.159 \mathrm{~cm}$. The depletion layer predicted by equations 1 to 3 extend $\mathrm{s} 0.06 \mathrm{~cm}$ away from the cathode. If the distance between the pie-pan plates were decreased by a factor of 3 , the ionization chamber would be nothing but depletion layer at late time. We are presently attempting to construct such a chamber.

\section{GENERAL DISCUSSION OF CONDUCTIVITY MEASUREMENTS}

Surface phenomena such as the ejection of secondary electrons due to the impact of ions on metallic surfaces are complicated and are greatly influenced by surface contamination. 11 It is difficult to interpret the behavior of a "pie-pan" ionization chamber in order to obtain information about one of the macroscopic parameters, such as electron conductivity, without knowing something about all of the other parameters that go into electron depletion layer formation. In a sense, when one interprets a "piepan" ionization measurement, one must not only rely on the validity of an air chemistry package involving many parameters, but also the accuracy of the model of the electron depletion layer.
Recent papers by workers at $\mathrm{HDL}^{19,20}$ describe Lband and $\mathrm{X}$-band microwave absorption measurements in the Aurora test cell designed to yield information about air conductivity and resolve apparent inconsistencies in the "pie-pan" air chemistry experiments. Theoretically, rf absorption measurements yield information about air conductivity, but there are other complications. One of these complications results from the fact that electron thermalization time can be of the same order as the rf period. Thus, it seemed more appropriate to pass an rf signal through a waveguide on which a much larger dc field is superimposed. In this case, the increase in electron temperature due to the rf probe signal can be negligible. Of course, if a dc field is imposed on a waveguide surface, an electron depletion layer is formed at the cathode.

The work described here represents our initial attempt to utilize a biased waveguide scheme to yield information concerning the field dependence of important air chemistry parameters. This technique does not avoid depletion layer complexities. A knowledge of the electron depletion layer behavior is still required to interpret the data. However, the biased waveguide technique yields an added independent experimental measurement that can be employed in the validation of a combined electron depletion layer--air chemistry package.

Car1 Baum ${ }^{21}$ has discussed some of the additional complexities of microwave absorption measurements in the presence of a large "dc" electric field. He derives an expression for the effective microwave conductivity using a perturbation analysis (for sufficiently small microwave electric fields) on the basic conductivity relationships. The validity of Baum's results require that, in the case of the probing $E-$ field parallel to the imposed E-field, the probing frequency always be much less than the electron energy exchange collision frequency. As will become clear, this limits his results to very low frequencies.

Baum's microwave conductivity matrix becomes quite simple if the matrix is expressed in terms of microwave electric field vector components parallel and perpendicular to the applied dc field vector. The conventional "dc" air conductivity can be expressed as

$$
\sigma(E)=e N \mu_{e}(E)
$$

where

and

$$
\begin{aligned}
e= & \text { the electron charge, } \\
N= & \text { the electron number density, } \\
\mu_{e}(E)= & \text { the electron mobility at field } \\
& \text { strength E. }
\end{aligned}
$$

If the ac probe frequency $w$ is much smaller than the momentum transfer collision frequency, $\nu_{m}$, the simple Lorentzian expression for the electron mobility $\mu_{\mathrm{e}}(\mathrm{E})$ can be expressed as

$$
\mu_{e}=\frac{e}{m v_{m}}
$$

where

$$
\mathrm{m}=\text { the electron mass. }
$$

The fractional energy (or temperature) loss per collision is given by $\nu_{\mu} / \nu_{\mathrm{m}}$ where $\nu_{\mu}$ is the electron energy transfer collision frequency. Baum assumes that $\omega \ll \nu_{\mu}$, where $\omega$ is the rf probe frequency, and obtains

$$
\left(\sigma_{\Delta}\right)=\left(\begin{array}{cc}
\sigma_{\mathrm{p}} & 0 \\
0 & \sigma_{\perp}
\end{array}\right)
$$


where $\sigma_{p}=\sigma(E)\left[1+\frac{E}{\mu} \frac{\partial \mu}{\partial E}\right]$ and $\sigma_{\perp}=\sigma(E)$ are the microwave conductivities when the microwave and $\mathrm{dc}$ electric fields are respectively parallel and perpendicular. $\sigma(E)$ is the conventional conductivity of ionized air (equations 4 and $4^{\prime}$ ).

At standard pressure and temperature and zero humidity, the momentum transfer collision frequency of thermalized electrons with air molecules is 21

$$
\nu_{\mathrm{m}} \simeq 1.1 \times 10^{11} \mathrm{sec}^{-1} \text {. }
$$

If the elastic sphere model is assumed to apply to air, the fractional energy loss or energy collision loss is 23

$$
\nu_{\mu} / \nu_{\mathrm{m}}=2 \mathrm{~m} / \mathrm{M} \approx 5 \times 10^{-5}
$$

and

$$
\nu_{\mu} \simeq 5 \times 10^{6} \mathrm{sec}^{-1} \text {. }
$$

Actually, the elastic sphere molecular model yields a lower limit for the electron energy exchange collision frequency and there are many inelastic collision phenomena which are not taken into consideration by the simple elastic sphere model. The ratio $\nu_{\mu} / \nu_{\mathrm{m}}$ is about $10^{-1}$ at high temperatures (electron kinetic energy about $20 \mathrm{eV}$ ) and about $10^{-3}$ at thermal temperatures. 22 The minimum frequency corresponding to $\nu_{\mu}$ at thermal temperatures is about

$$
\mathrm{f}=\frac{10^{-3} \nu_{\mathrm{m}}}{2 \pi}=18 \text { megacycles }
$$

and in the case of the elastic sphere model the frequency is approximately 1 megacycle. This frequency places a severe limitation on the applicability of expression 5 .

The simple Lorentz dc conductivity expression ( $\omega$ $\left.<<\nu_{m}\right)$ given by equation 4 can be compared to a more general expression based on the Boltzmann equation: ${ }^{23}$

$$
\sigma=-\frac{4 \pi n e^{2}}{3 m} \int \frac{1}{v(v)} \frac{d f(v)}{d v} v^{3} d v
$$

where $f(v)$ is the electron velocity distribution function. In expression 6 the collision frequency $v(v)$ is a function of velocity, and the conductivity is obtained by integrating over all velocities. The velocity distribution function $f(v)$ is a function of the electron temperature $\mathrm{U}_{\mathrm{e}}$. In his derivation of equation 5, Baum assumes that the value of the ac probe frequency is low enough for the distribution function $f(v)$ to be in equilibrium with energy changes introduced by the ac probe.

In the L-band measurements to be discussed in the next section, $\omega=2 \pi 1.5 \times 10^{9}$ radians $/ \mathrm{sec}$, a frequency larger than the energy collision loss frequency. The significance of this high frequency must be examined; specifically the variation of the electron temperature during an $\mathrm{rf}$ cycle can be estimated in a manner similar to the method described in reference 22. It can be shown, using arguments similar to those presented in reference 22, that the maximum deviation of the electron temperature $U_{e}$ when an $r f$ probe voltage $\left(E_{0} \cos \omega t\right)$ is superimposed on a dc bias, $E$ is given by

$$
\pm \Delta U_{e}=\frac{4}{3} \frac{e}{m} \frac{E^{2}}{\nu_{m}}\left(\frac{E_{0}}{E}\right) \frac{1}{\omega} .
$$

In reference 22 Baum gives the following estimates of $U_{e}, \nu_{\mu}$, and $\nu_{m}$ for a dc bias $(E=10,000$ volt $\mathrm{s} / \mathrm{m})$ in air with $1 \%$ water content:

$$
\begin{aligned}
\mathrm{U}_{\mathrm{e}}= & 8.0 \times 10^{-2} \mathrm{eV} \quad, \mathrm{U}_{\mathrm{o}}=2.4 \times 10^{-2} \mathrm{eV}, \\
\nu_{\mu} & =10^{9} \mathrm{cycles} / \mathrm{sec}, \\
\nu_{\mathrm{m}} & =5 \times 10^{11} \mathrm{cycles} / \mathrm{sec} ;
\end{aligned}
$$

If the magnitude of the $\mathrm{rf}$ probe voltage is \pm 50 volts, then

$$
\begin{aligned}
& \frac{\mathrm{E}_{\mathrm{o}}}{\mathrm{E}}=\frac{50}{10,000}=5.0 \times 10^{-3} \\
& \Delta \mathrm{U}_{\mathrm{e}}=2.5 \times 10^{-5} \mathrm{eV} .
\end{aligned}
$$

and

It is, therefore, reasonable to assume that the electron temperature is relatively constant. The values for $E$ and $E_{O}$ used here correspond to the L-band experiments described in the next section of this report.

\section{BIASED L-BAND WAVEGUIDE MEASUREMENTS}

A first-generation biased L-band waveguide was designed to enhance the scientific utility of $\mathrm{rf}$ probes over a wide range of electric field strengths. Another consideration is that the information obtained in a combined ionization-rf measurement would provide insight into the behavior of an ionzied conducting gas near a negatively biased metallic conductor.

As already indicated, the L-band data to be presented in this work correspond to a situation in which the low power ( $\left(s m a 11 \mathrm{E}_{\mathrm{o}}\right.$ ) rf probe frequency is greater than the energy exchange frequency; hence, the anisotropy effects derived by $\mathrm{Baum}^{21}$ are not pertinent to these measurements. Also, the electron temperature is not significantly changed during an $\mathrm{rf}$ period (equation 9).

A biased L-band waveguide device is shown in figure 7. The purpose of the experimental arrangement is to obtain information about the conductivity of ionized air with an $\mathrm{rf}$ probe in addition to the information obtained by analyzing dc pie-pan-like currents. The perturbation of the wave guide was minimized in order that the reflection due to the bias plate could be ignored.

Neglecting depletion layer effects, the dc field in the biased waveguide is as shown in figure 8 . If an appreciable field perturbation due to electron depletion layer formation is present, the analysis of our data becomes a relatively complicated, but soluble problem. A number of typical absorption measurements obtained with the biased waveguide with different plate bias voltages are shown in figure 9. The measured ratios of electron mobility to the electronoxygen attachment rate derived from absorption measurements (such as shown in figure 9) are shown as circles in figures 10 and 11 . The figures also show results computed from air chemistry packages proposed by other workers in the EMP community. $3,8,9,10$.

The E-field in the biased waveguide measurements has been varied over two orders of magnitude: $10^{5}$ volts/meter to 800 volts/meter. Up to now we have only determined the ratio of the electron mobility to the electron attachment rate, $\mu / \alpha$. These measurements have an accuracy of about $\pm 20 \%$ and will be improved as our dosimetry techniques are enchanced. (Note that the ratio of mobility to attachment rate predicted by the various air chemistry packages employed in the EMP community yield values that can differ by as much as $100 \%$ ). 
There is no reason why the electric bias field cannot be varied over a much greater range than that shown in figures 10 and 11 . The technique can also be employed at different frequencies, at reduced air or gas pressure, and at different percentages of water vapor. Electron mobility and the electron attachment rate to $\mathrm{O}_{2}$ are strongly dependent on air pressure. Electron absorption measurements at reduced pressure should yield independent values of the mobility $\mu$ and the attachment rate $\alpha$. Preliminary measurements at 0.1 atmosphere indicate that electron absorption measurments at reduced pressure can yield independent values of these two parameters.

\section{SUMMARY AND CONCLUSIONS}

We have investigated ways to measure the conductivity of gases (air and nitrogen) in the presence of a pulsed ionizing radiation source and imposed electric fields. The approaches included simple dc measurements using a "pie pan" type chamber and microwave absorption measurements.

Analyses of the experimental data are complicated by the formation of an electron depletion region at the surface of the negatively charged metallic conductor. The effects of electron depletion (and concomitant electric field enhancement) near the cathode are more easily studied in nitrogen than in air due to the rapid attachment of conduction electrons to oxygen molecules. The data suggest that for nitrogen gas the late-time conductivity (after the radiation source has terminated) in our experiments was in part due to collision induced secondary electrons emitted from the cathode surface. Such phenomena are complex, being strongly dependent on surface conditions, and, hence, quantitative analyses of the data are uncertain. A 0.5 percent probability of secondary emission resulting from ionic impact provided a good fit to the measured data.

Figure 12 is a simple circuit diagram that can be employed to understand both a conventional pie-pan measurement and a biased waveguide rf absorption measurement. The neutral ionized region in an ionization chamber or a biased waveguide can be represented with a resistance, $\mathrm{R}_{1}=\mathrm{Al}_{1} / \sigma_{1}$, where $\mathrm{A}$ is the effective cross sectional area of the pie-pan or waveguide, $\ell_{1}$ the distance across the pie-pan, and $\sigma_{1}$ the conductivity of the region. The electron depletion layer consists of a resistor of the same cross section $A$, of length $\ell_{2}$, and conductivity $\sigma_{2}$. If there is no secondary electron emission at the cathode, $\sigma_{2}$ corresponds to pure ionic conductivity. A pie-pan measurement determines the current flow through a circuit consisting of $R_{1}$ and $R_{2}$ in series. The magnitude of the absorption in a biased waveguide measurement yields an independent determination of the conductivity, $\sigma_{1}$ (see equations 4 and $4^{\prime}$ ). Both measurements are interpreted using equations 1 to 3 plus an air chemistry package.

Air chemistry reaction rates are important microscopic parameters that help determine the behavior of an infinite expanse of ionized air. Conduction currents flowing between a metallic system and ionized air play an important role in the interaction of a system with a nuclear source region. One must know more than reaction rates in order to understand these currents. The determination of the conductivity of an infinite expanse of ionized air with no boundary conditions does not supply enough information to determine the electron flow through a metallic object perturbing this expanse.

\section{REFERENCES}

1. W. F. Crevier, C. L. Longmi re, G. Merke1, and D. Spohn, "Air Chemistry and Boundary Layer Studies with Aurora," IEEE Trans. Nucl. Sci., NS-24, No. 6,1977 , p. 2046.

2. M. L. Price and V. A. J. Van Lint, "Measurement of Electron Attachment and Mobility in Dry and Wet Air," Mission Research Corporation, San Diego, CA, Defense Nuclear Agency, DNA-4788/T, December 1978.

3. E. Pettus, W. F. Crevier, "Analytic Representation of Electron Mobility and Attachment Data in Dry and Moist Air from Van Lint's HIFX Experiments," Mission Research Corporation, MRC-R-576, July 1980 .

4. C. L. Longmi re, "The Boundary Layer in Close-In EMP Coupling," Aurora EMP Memo 5, October 1976.

5. R. H. Fowler and L. Nordheim, Proc. R. Soc. Al 19, 1928, p. 173.

6. W. Z. Schottky, Phys. 14, 1923, p. 63.

7. R. W. Strayer, F. M. Charbonnier, E. C. Cooper, and L. W. Swanson, Field Emission Corporation Quarterly Report No. 3, (1 Nov. 1962 to 31 Jan. 1963).

8. H. J. Longley, and C. L. Longmi re, "Electron Mobility and Attachment Rate in Moist Air," Mission Research Corporation, Santa Barbara, CA, MRC-N-222, December 1975.

9. W. F. Crevier and C. L. Longmire, "EMP Air Chemistry Experiments with Aurora," Aurora EMP Memo 4, March 1976.

10. W. Radasky, "An Examination of the Adequacy of the Three-Species Air Chemistry Treatment for the Prediction of Surface-Burst EMP," MRC, ADA025 280, December 1975.

11. Ear1 W. McDaniel, "Collision Phenomena in Ionized Gases," John Wiley and Sons, Inc., New York, 1964, Chapt. 13.

12. J. H. Parker, Phys. Rev. 93, 1954, p. 1148.

13. Robert N. Varney, "Liberation of Electrons by Positive-Ion Impact on the Cathode of a Pulsed Townsend Discharge Tube," Phys. Rev., 93, 1954, p. 1156.

14. H. S. W. Massey and E. H. S. Burhop, "The Co11ision of Positive Ions and Neutral Atoms with Surfaces," Chapter IX in Electronic and Ionic Impact Phenomena, Oxford University Press, Great Britain, 1956.

15. H. D. Hagstrum, Phys. Rev. 104,1956, p. 1516.

16. H. D. Hagstrum and C. D'Amico, J. App1. Phys. 31,1960 , p. 715 .

17. H. D. Hagstrum, Phys. Rev, 89,1951 , p. 244.

18. R. H. Good and E. W. Mul $\overline{l e r}$, Enclyclopedia of Physics, 21, 1956, p. 176.

19. L. Ambrose, M. Bushe11, C. Kenyon, G. Merke1, W. D. Scharf, "Microwave Measurements on Conductivity in Air and Other Gases," IEEE Trans. Nucl. Sci., NS-28, No. 6, Dec. 1981, p. 4426.

20. L. Ambrose, M. Bushe11, E. Hirschorn, C. Kenyon, G. Merke 1, and W. D. Scharf, "Determination of the Ionic Conductivity of Ionized Air with a Resonant Cavity," IEEE Trans. Nuc1. Sci., NS-30, No. 6, Dec. 1983 , p. 4577.

21. C. E. Baum, "Some Limitations on Microwave AirConductivity Measurements," Note 16, in Electromagnetic Pulse Sensor and Simulation Notes, AFWL EMP 1-1, September 30, 1965.

22. C. E. Baum, "Electron Thermalization and Mobi1ity in Air," Note XII in Electromagnetic Pulse Theoretical Notes, Vo1. 1, DASA $1882-1$, July 16 , 1965.

23. M. A. Heald and C. B. Wharton, "Plasma Diagnostics with Microwaves," John Wiley and Sons, Inc., New York, 1965, p. 60. 


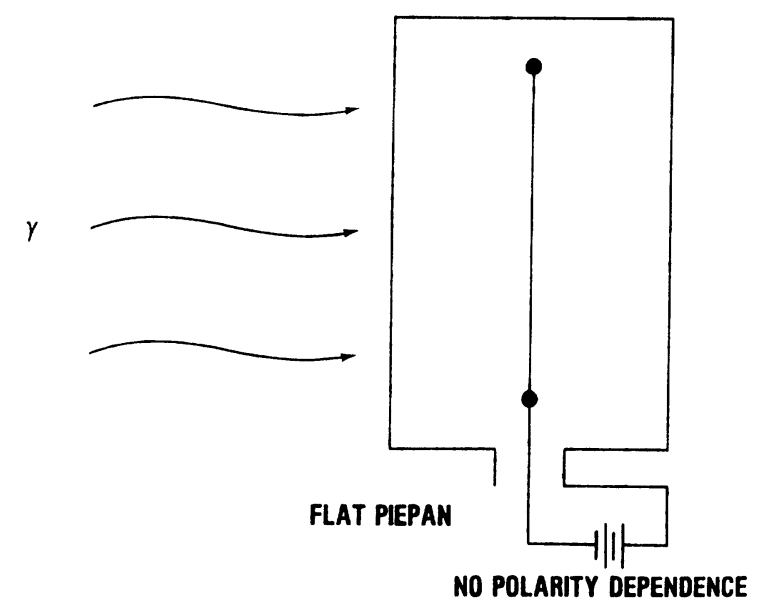

Figure 1. Schematic diagram of one-dimensional flat "pie-pan".

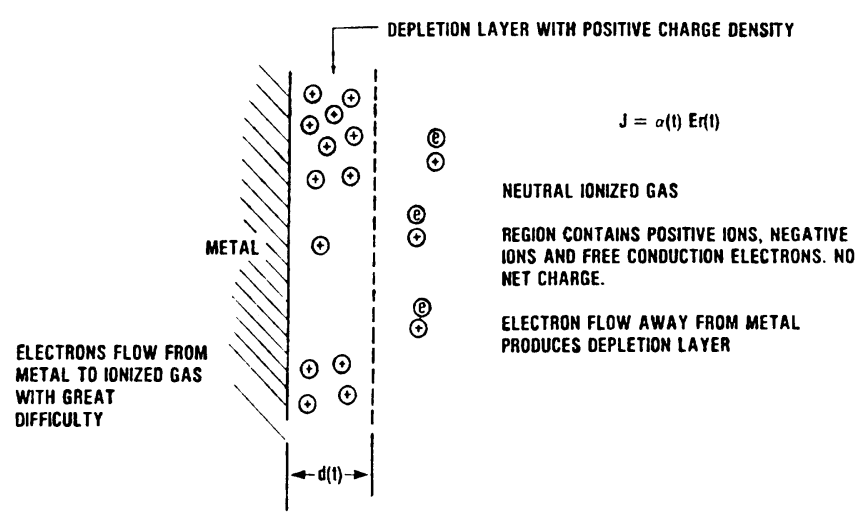

Figure 2. Schematic diagram of electron depeletion layer.

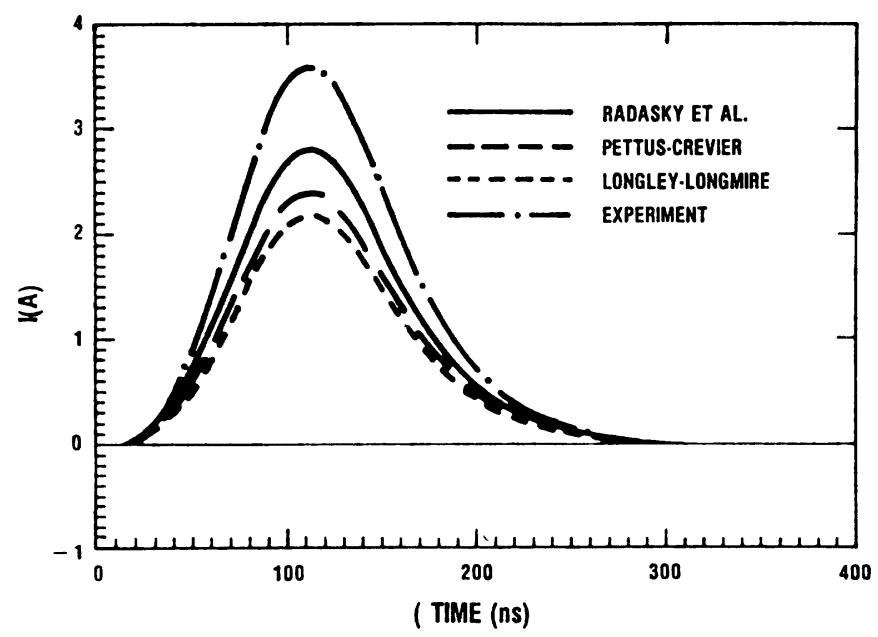

Figure 3. Finite-difference code predictions of current response of $1 / 8$ inch pie-pan using various air-chemistry packages. Experimental Results are also shown. Bias was 500 volts.

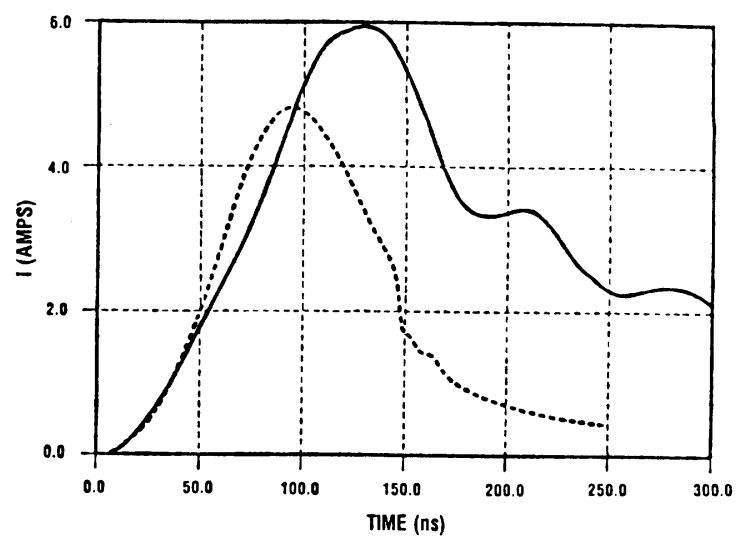

Figure 4. Comparison between experimental pie-pan measurement (solid line), and finitedifference calculation (dot-dot): nitrogen, 1000 volts, 300 rads.

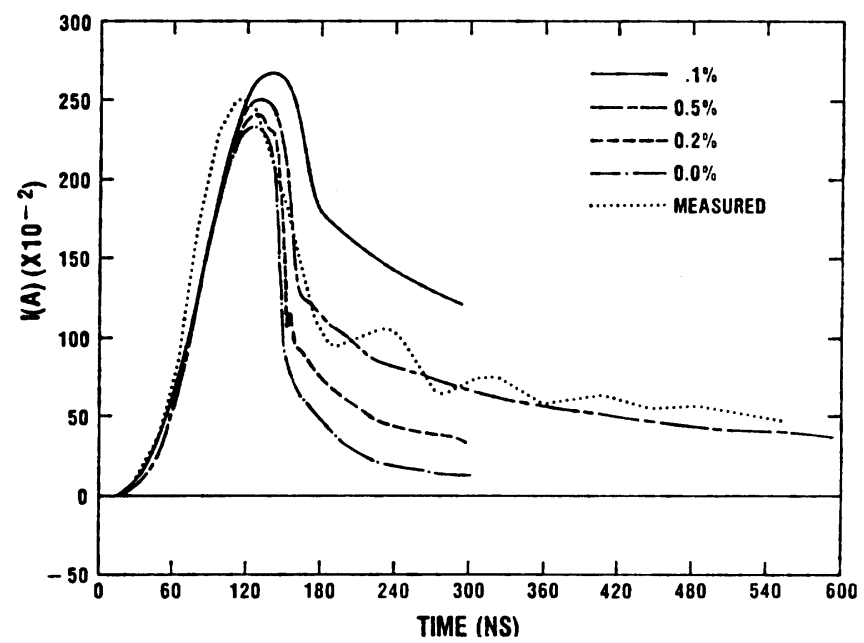

Figure 5. Comparison of experimental pie-pan ionization chamber measurement (200 volts) with calculations corresponding to 4 different probabilities for secondary electron emission due to ion impact.

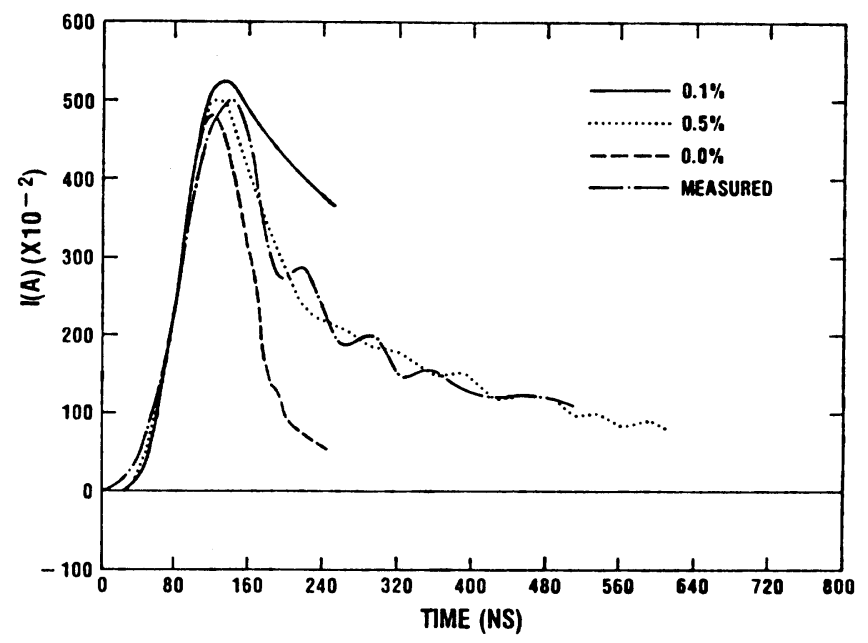

Figure 6. Comparison of experimental pie-pan ionization chamber measurement $(1,000$ volts) with calculations corresponding to 3 different probabilities for secondary electron emission due to ion impact. 
WAVEGUIDE

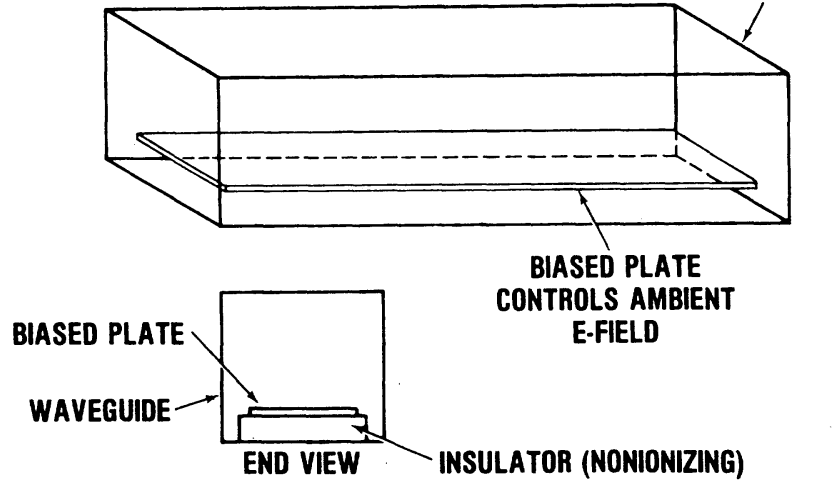

Figure 7. Sketch of biased L-band waveguide.



Figure 8. Distribution of equipotential surfaces in biased L-band waveguide.

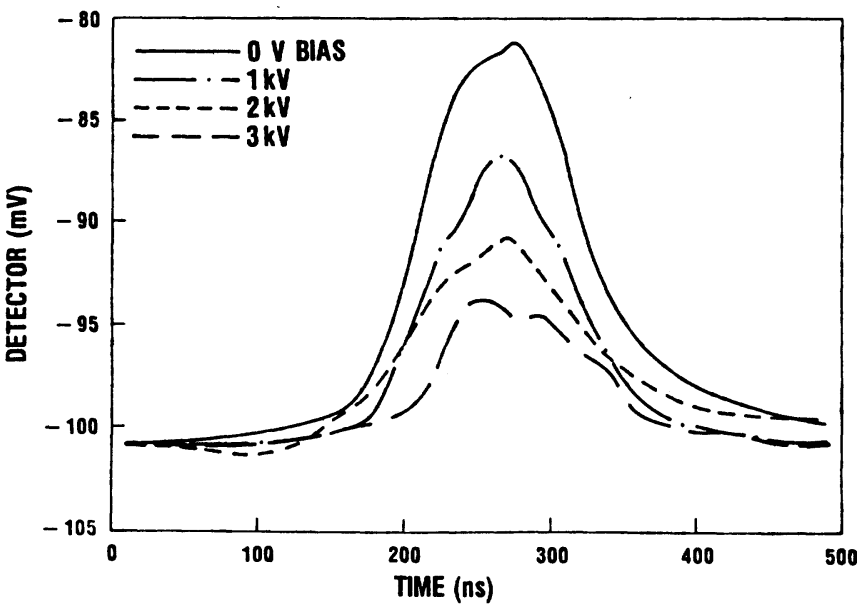

Figure 9. Detector response for dry air in biased Lband waveguide.

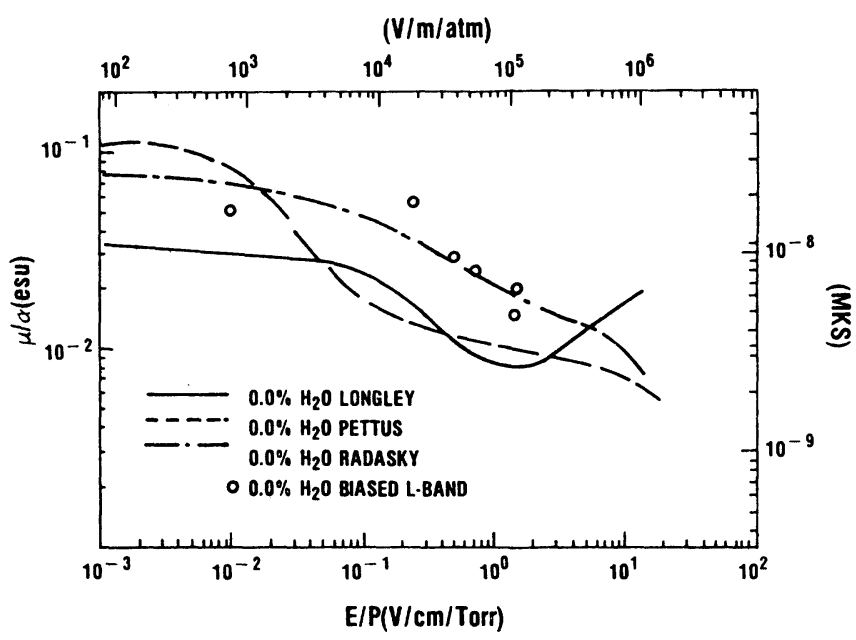

Figure 10. Values of the ratio of the electronic mobility to the electronic attachment coefficient obtained with the biased Lband $\mathrm{rf}$ absorption technique. The water content of the air was $1 \%$.

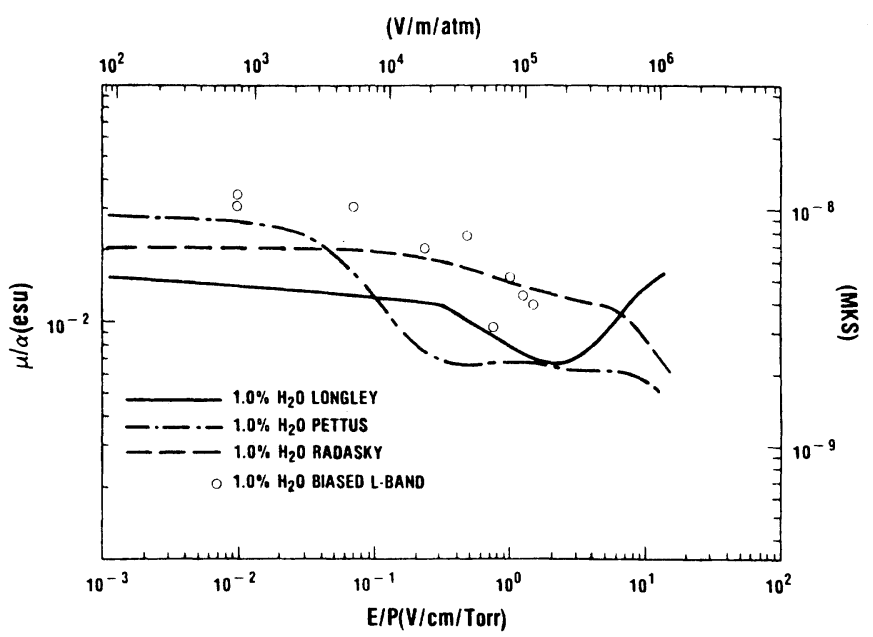

Figure 11. Values of the ratio of the electronic mobility to the electronic attachment coefficient obtained with the biased Lband $\mathrm{rf}$ absorption technique. The water content of the air was $0 \%$.

$$
R_{1}=A\left(l_{1} / \sigma_{1}\right) \quad R_{2}=A\left(l_{2} / \sigma_{2}\right)
$$

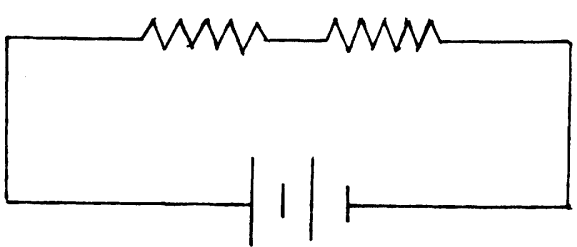

Figure 12. Simple circuit model of ionization chamber of area $A ; \delta_{1}=$ distance across non-electron depletion region, $\sigma_{1}=$ conductivity of non-electron depletion region, $\AA_{2}=$ distance across electron depletion region, and $\sigma_{2}=$ conductivity of electron depletion region. 